University of Wollongong

Research Online

Faculty of Informatics - Papers (Archive)

Faculty of Engineering and Information

Sciences

January 2006

\title{
Adaptive resource replication in a ubiquitous peertopeer based multimedia distribution environment
}

\author{
L. Rong \\ University of Wollongong \\ I. Burnett \\ University of Wollongong, ianb@uow.edu.au
}

Follow this and additional works at: https://ro.uow.edu.au/infopapers

Part of the Physical Sciences and Mathematics Commons

\section{Recommended Citation}

Rong, L. and Burnett, I.: Adaptive resource replication in a ubiquitous peertopeer based multimedia distribution environment 2006.

https://ro.uow.edu.au/infopapers/257

Research Online is the open access institutional repository for the University of Wollongong. For further information contact the UOW Library: research-pubs@uow.edu.au 


\title{
Adaptive resource replication in a ubiquitous peertopeer based multimedia distribution environment
}

\author{
Abstract \\ A dynamic P2P architecture was proposed in our previous work to support resource adaptation/ \\ personalization according to the surrounding usage environment and user preferences. In this paper, we \\ propose an adaptive resource replication strategy based on the proposed P2P architecture. It uses \\ resource request rate as the metric to trigger the resource replication process, and proportionally \\ replicate multimedia resources into various configuration states according to the properties of peers (i.e., \\ terminal capabilities and user preferences) and the size of peer clusters. Also, the strategy uses peer \\ related information stored on super peers to determine which peers should be selected to perform \\ adaptive replications and where the resulting replicas should be stored. Simulation results show that the \\ proposed strategy reduces network delays while increasing resource hit rate in comparison to FreeNet \\ and random replication strategies.

\section{Disciplines} \\ Physical Sciences and Mathematics

\section{Publication Details} \\ This paper originally appeared as: Rong, L \& Burnet, I, Adaptive resource replication in a ubiquitous \\ peertopeer based multimedia distribution environment, 2006 3rd IEEE Consumer Communications and \\ Networking Conference, 8-10 January 2006, vol 1, 65-68. Copyright IEEE 2006.
}




\title{
Adaptive Resource Replication in a Ubiquitous Peer- to-Peer based Multimedia Distribution Environment
}

\author{
Letian Rong, Ian S Burnett \\ School of Electrical, Computer and Telecommunication Engineering, \\ University of Wollongong \\ Wollongong, Australia \\ 1r98@uow.edu.au, i.burnett@elec.uow.edu.au
}

\begin{abstract}
A dynamic P2P architecture was proposed in our previous work to support resource adaptation/personalization according to the surrounding usage environment and user preferences. In this paper, we propose an adaptive resource replication strategy based on the proposed P2P architecture. It uses resource request rate as the metric to trigger the resource replication process, and proportionally replicate multimedia resources into various configuration states according to the properties of peers (i.e., terminal capabilities and user preferences) and the size of peer clusters. Also, the strategy uses peer related information stored on super peers to determine which peers should be selected to perform adaptive replications and where the resulting replicas should be stored. Simulation results show that the proposed strategy reduces network delays while increasing resource hit rate in comparison to FreeNet and random replication strategies.
\end{abstract}

\section{INTRODUCTION}

The rapid spread of Internet-based P2P applications such as Napster [6] has inspired the research and development of technologies for P2P services and systems. Much of the attention has been focused on the issues of providing scalability, interoperability or routing mechanisms within P2P networks. However, as computing devices become more diverse, it is important that $\mathrm{P} 2 \mathrm{P}$ systems provide resource sharing suitable for a ubiquitous computing environment. In particular, resources should be adapted to suit the usage environment of the consuming terminal device and thus provide the user with the best possible experience [7]. A super peer-based architecture was proposed in our previous work [8] to facilitate dynamic resource adaptation in a P2P environment based on the surrounding usage environment attributes (i.e., user preferences, terminal capabilities etc,).

In this paper, we focus on the resource replication aspect of the proposed architecture. As the proposed architecture aims to provide a $\mathrm{P} 2 \mathrm{P}$ infrastructure enabling multimedia access in a ubiquitous computing environment, the replication strategy needs to take the heterogeneity of peers into consideration when creating resource replicas. In addition, it should also use the properties of peers (i.e., terminal capabilities) and their surrounding usage environment attributes (i.e., network attributes) to determine which peers should be selected to perform adaptive replications and where the resulting replicas should be stored. This is an area that has not been explored, to our knowledge, in other work.
The paper is structured as follows: section 2 compares the related work in the field, outlining the shortcomings and challenges currently being faced. Section 3 briefly describes the proposed dynamic resource adaptation architecture and then in section 4 the proposed resource replication strategy is explained. The simulation results are shown in section 5 with section 6 drawing conclusions.

\section{RELATED WORK}

$\mathrm{P} 2 \mathrm{P}$ based replication strategy is the topic of various active research projects and most of these projects emphasize replication in a structured $\mathrm{P} 2 \mathrm{P}$ network $[2,3,5]$. There are several projects on replication in unstructured $\mathrm{P} 2 \mathrm{P}$ networks; in particular, Lv et al [1] studies optimal replication in order to reduce random search times in an unstructured P2P network. Two common replication methods: Uniform and Proportional replication strategies were considered in the work, and a third replication strategy: Square-root replication was then proposed to provide the optimal search size on successful content request queries and minimize the overall search traffic. In [4], a set of Quality of Service (QoS) based parameters and metrics are proposed to evaluate the performance of content replication in P2P network. It focuses on P2P systems where peer storage is very limited and uses replica placement as the main strategy to improve content replications by satisfying the proposed QoS parameters.

One important missing aspect of the projects above is that they do not consider the heterogeneity of a P2P environment where replications should be adaptively created in proportion to the percentage of various peer classes (i.e., desktop computer, mobile phone etc.) and based on their usage environment factors (i.e., terminal capabilities). For instance, a movie trailer should be replicated in various bit-rates/resolutions so that it can be played on not only desktop computers, but PDAs and mobile phones which all connect to the same P2P network. Also, the mentioned projects take a passive replication approach which only triggers the replication process when a resource is downloaded by a peer and creates a maximum of one resource replica. We argue that it is essential to provide a more assertive replication strategy to cope with the dynamic changes of resource popularity in a multimedia sharing P2P network. The following work concentrates on the design of a replication strategy based on a dynamic resource adapting P2P architecture. 


\section{DyNAMiC RESOURCE AdAPTATION P2P System}

In our previously proposed dynamic resource adaptation $\mathrm{P} 2 \mathrm{P}$ architecture [8], peers are grouped into clusters based on similarity of their shared contents and category super peers (i.e., super peers who govern one particular type of content) are nominated to be in charge of one or more clusters. On joining the network, a peer must submit information about its shared resources and its related usage environment attributes to the category super peer(s). Category super peers then use the registered information to adapt search results and select peers to send/adapt resources to the request peers.

The proposed architecture was then further improved through a locality-based clustering approach which assigns peers to locality clusters based on their locality information [10]. Therefore, peers are clustered according to not only their shared contents but also their locality information. The approach reduces network delays through locating resources which are nearby to the request peers (i.e., resources in the same or nearby locality clusters). The proposed adaptive resource replication strategy in this paper was designed based on the improved system.

\section{AdAPtive Resource ReplicAtion Strategy}

Based on the observations in Section 2, we propose a novel adaptive resource replication strategy that improves the resource availability and reduces network delays. In order to accommodate dynamic changes of resource popularity in a P2P network, we take a more assertive replication approach by using request rate as the metric to determine when replication should be performed. The request rate is calculated as the number of requests received by the category super peer for a particular resource divided by the time elapsed.

Each category super peer has a request rate table which contains entries that store both the total number of requests and request rates of resources that belong to the category; the table is updated every time when a category super peer receives a resource request. When the total number of requests for a resource reaches a certain predefined threshold (i.e., every 20 requests), it triggers the category super peer to check if resource replication for the particular resource is required. Presently, the following simple formula is used to determine the number of replicas (Required $_{\text {Total }}$ ) that are required globally in the network for a given request rate of a resource (Request_Rate):

$$
\text { Required }_{\text {Total }}=\text { Request_Rate } * K
$$

$K$ is a numeric value that determines how aggressively the replication strategy should be undertaken. If the total number of current resource variations is smaller than Required $_{\text {Total, }}$, then the super peer starts the adaptive resource replication process.
The replication process is divided into four steps:

Step1. Overall replica number estimation for each locality cluster: the category super peer estimates the number of resources that are required to be replicated in each locality cluster based on Required Total $_{\text {and }}$ ane size of each locality cluster $\left(\right.$ Size $\left._{\text {Cluster }}\right)$ in comparison to the total network size $\left(\right.$ Size $\left._{\text {Total }}\right)$. For instance, the required number of resources in locality cluster i (Required $\left._{\text {Cluster-i }}\right)$ is calculated as:

$$
\text { Required }_{\text {Cluster-i }}=\text { Required }_{\text {Total }} *\left(\text { Size }_{\text {Chuster }} / \text { Size }_{\text {Total }}\right)
$$

The category super peer then requests each locality super peer to replicate Required $_{\text {Cluster }}$ number of replicas.

Step2. Individual replica number estimation based on each resource variation: once a locality super peer receives the order from the category super peer, it starts the second stage of the replication process by calculating the number of replicas that are required for each resource variation (i.e., resource with different configuration state). As explained in section 3, every peer is required to register its related usage environment descriptors (i.e., terminal capabilities and user preferences) to a corresponding locality super peer; therefore the locality super peer uses the peer information to calculate the number of peers $\left(\right.$ Size $\left._{\text {Peer-Variaiton }}\right)$ which fit a resource variation. For instance, a movie trailer with resolution of $320 \times 240$ is suitable for peers running on PDAs. The required number of replicas for resource variation A (Required $\left._{\text {Variation-A }}\right)$ in cluster i of size Size $_{\text {Cluster- } i}$ is then calculated as:

$$
\begin{gathered}
\text { Required }_{\text {Variation-A }}=\text { Required }_{\text {Cluster-i }} * \\
\left(\text { Size }_{\text {Peer-Variaiton-A }} / \text { Size }_{\text {Cluster-i }}\right)
\end{gathered}
$$

Step3. Allocate replica senders, adaptors and receivers: when Required $_{\text {Variation }}$ is determined for each resource variation in a locality cluster, the locality super peer selects replica senders, adaptors and receivers respectively. We define replica adaptors as the peers able to adapt a resource to a different resource variation (i.e., transcoding of video streams). The replica senders, adaptors and receivers are selected based on their capability descriptors (i.e., bandwidth, processing power, storage capacity and availability etc.,) which are stored on the locality super peer. The selection process is the same as the peer sender (i.e., peer which sends/adapts a request resource) selection process proposed in [8] and it continues until Required $_{\text {Cluster }}$ is reached in the locality cluster. This selection process occurs in each locality cluster and the adaptive replication process is completed when all replicas are transmitted to the replica receivers in every locality clusters.

Step4. Resource replacement strategy: the resource replacement strategy is deployed when the storage capacity of a peer has reached its maximum and some resources stored on the peer are required to be removed to create space for the 
resource replica. The peer retrieves request rates of stored local resources from corresponding category super peers and removes resource(s) with the lowest request rate(s) until enough space is freed for a new replica.

\section{Simulation Results}

A simulation was created in our work [8] to evaluate the performance of the proposed dynamic resource adapting architecture. We added code to the simulation based on the proposed adaptive resource replication strategy to evaluate its performance.

TABLE I. SIMULATION SETTINGS

\begin{tabular}{|l|l|}
\hline Total no. of peers & 3000 \\
\hline No. of super peers (including locality super peers) & 60 \\
\hline No. of locality super peers & 30 \\
\hline No. of provider peers & 240 \\
\hline No. of freeloaders & 2700 \\
\hline No. of unique resources (initially during startup) & 500 \\
\hline Resource size & $10-200 \mathrm{Mbs}$ \\
\hline Percentage of total resources owned by freeloaders & $30 \%$ \\
\hline $\begin{array}{l}\text { Percentage of total resources owned by provider } \\
\text { peers/super peers }\end{array}$ & $70 \%$ \\
\hline Zipf skew factor & 0.271 \\
\hline No. of new peers joined the network while running & 1000 \\
\hline No. of peers left the network while running & 1000 \\
\hline Total no. of requests & 10000 \\
\hline
\end{tabular}

Initialization of the simulation involves preloading the network with a specified number of normal peers, locality super peers, other category-based super peers and resources. Normal peers are further classified into provider peers and freeloaders. A predefined number of unique resources are categorized, ranked (i.e., the most popular resource has a rank of one and so forth), populated and assigned to randomly selected normal peers according to the Zipf distribution. The normal peers are then assigned to locality super peers and other category-based super peers according to their randomly generated location attributes and the shared resource contents respectively. To simulate heterogeneous devices, three classes of device with different processing power, storage capacity and bandwidth are currently used in the simulation (i.e., high-end, normal and low-end peers). These system settings and peer behaviors conform to the findings in the survey from [9]. Furthermore, network delay is introduced in the network. Requests are generated by peers in the system at random time intervals based on the popularities of resources and the simulation terminates when all requests are completed.

Furthermore, several major improvements have been made to the simulation to create a more realistic P2P simulation environment. These include allowing a predefined number of randomly generated/selected peers with resources to join/leave the network at random intervals. Also, the popularities of old resources gradually decrease during the simulation.

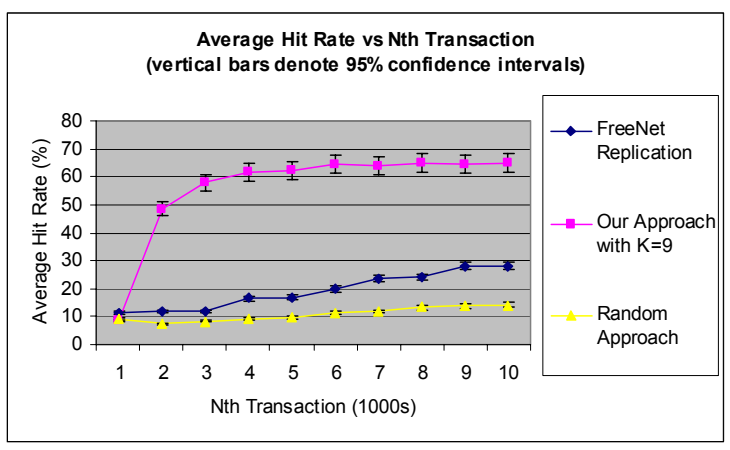

Figure 1. Average hit rates of different approaches

The results below were obtained based on the simulation settings in Table 1. We have also simulated other peer compositions, and the simulation results shown in the section demonstrate the typical trend.

In the simulation, we compare the proposed replication strategy with replication in FreeNet [11] and a random replication strategy. FreeNet always makes a replica for each requested resource. The request peer tries to store a new resource into the local storage. However, when there is not enough storage space for the new resource, a Least Recent Used (LRU) replacement policy is used to remove old contents. In the random replication approach, the requested content is randomly assigned (i.e., yes or no) to be replicated into the local storage.

Average hit rate is used as a performance metric to measure the performance of the proposed replication strategy. The average hit rate is calculated based on the percentage of times a required resource is available in the locality cluster of the request peer.

Fig. 1 shows that when $K$ is set to 9 , the hit rate increases by 3-4 times in comparison to the FreeNet Replication strategy and 4-5 times in comparison to the random replication approach. The increase of resource availability also contributes to the decrease of average network delay which is evident in Fig. 2, where our proposed approach outperformed the FreeNet replication strategy and random replication approach by 30 $50 \%$ and $60-70 \%$ respectively. Also, our approach shows a higher network delay in comparison to the FreeNet replication strategy in the beginning of the simulation, but quickly drops below it after the first 1000 requests.

The second comparison is made between the proposed replication strategy and a modified replication strategy which follows step1 of Section 4, but replaces step2 with choosing resource variations randomly to replicate in each locality clusters. Average download speed is used as a performance metric and it shows in Fig. 3 that the proposed approach has a $10-15 \%$ higher download speed than the modified replication strategy. 


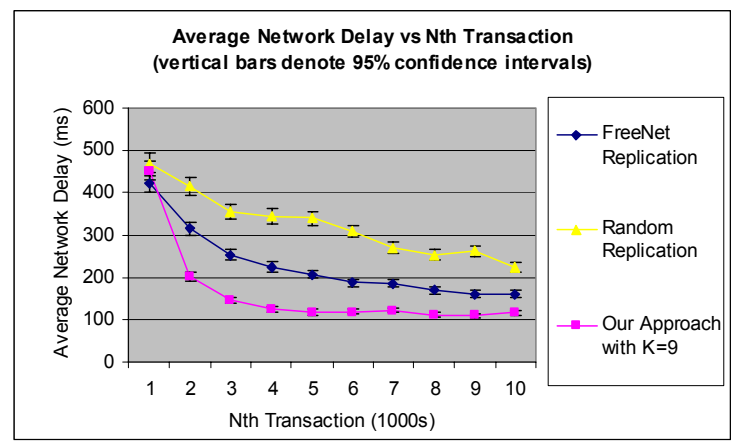

Figure 2. Average network delay of different approaches

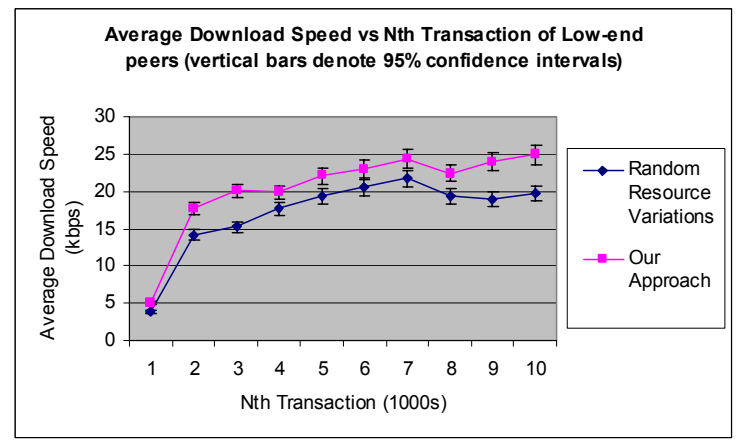

Figure 3. Average download speed of low-end peers of different approaches

Next, we vary the $K$ value of the proposed approach and compare the results. Fig. 4 shows that the hit rate increases steadily as the value of $K$ increases, until it reaches beyond a value of 9; after that, the hit rate does not show evident improvements; this is revealed in the graph when the $K$ is set to 15. Simulation results for other peer compositions also indicate that the $K$ value has a threshold.

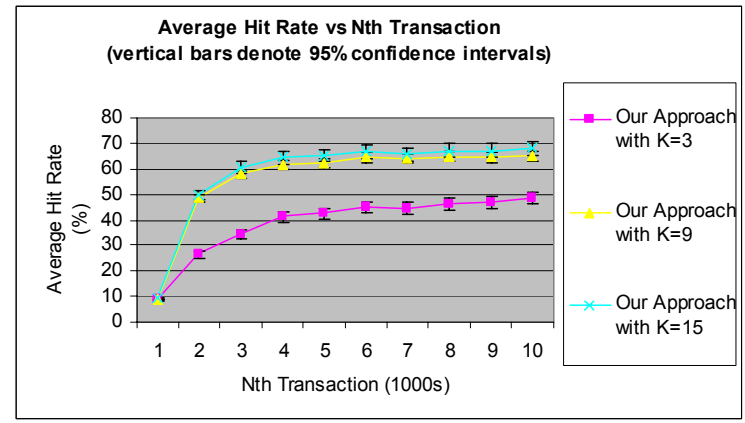

Figure 4. Average hit rate of the proposed replication strategy with different $K$ value

Finally, the simulation also shows that by deploying the proposed replacement strategy as described in step 4 of Section 4 , the number of resources in the network is sustained at a certain level (i.e., when $K$ is set to 3 and 9 , there is a $4 \%$ and $12 \%$ increase in number of resources respectively) while retaining the average hit rate performance and keeping the average network delay low as mentioned above.

\section{CONCLUSION}

In this paper, we have proposed an adaptive resource replication strategy to facilitate resource replication in a dynamic resource adaptation P2P network. The replication strategy takes an active approach through using resource request rate as the prime metric to trigger the replication process and then adaptively creating resource variations in the network according to the heterogeneities of peers. Simulation results have shown that the proposed replication strategy could greatly improve the average hit rate of resources while keeping the average network delays low in comparison to the replication in FreeNet or a random replication approach. Also, the results show that replicating resources into resource variations in proportion to the heterogeneities of peers improves the average download speed, compared to the random resource variation replication approach. Furthermore, the replacement strategy maintains the total number of resources in the network at a reasonable level.

In our future work, intend to derive a formula to calculate the optimum values (i.e., hit-rate, download speed) in the proposed architecture and use it to further evaluate the performance of the proposed replication strategy.

\section{ACKNOWLEDGMENT}

The authors would like to acknowledge the support of the Smart Internet Technology CRC.

\section{REFERENCES}

[1] Q. Lv, P. Cao, E. Cohen, K. Li and S. Shenker, "Search and Replication in Unstructured Peer-to-Peer Networks", in ACM International Conference on Supercomputing 2002, New York City, USA, June, 2002.

[2] Z. Xiang, Q. Zhang, and W. Zhu, "Replication Strategies for Peer-toPeer Based Multimedia Distribution Service", in IEEE ICME'03, Baltimore, Maryland, USA, July, 2003.

[3] J. Kangasharju, K. W. Ross, and D. A. Turner, "Optimal Content Replication in P2P Communities", Manuscript, 2002.

[4] G. On, J. Schmitt, and R. Steinmetz, "the Effectiveness of Realistic Replication Strategies on Quality of Availability for Peer-to-Peer Systems", in Third International Conference on Peer-to-Peer Computing ( $\left.P 2 P^{\prime} 03\right)$, Linkoping, Sweden, September, 2003.

[5] Z. Zhang, and Q. Lian, "Reperasure: Replication Protocol Using Erasure-Code in Peer-to-Peer Storage Network", in $21^{\text {st }}$ Symposium on Reliable Distributed Systems, Suita, Japan, October, 2002.

[6] T. Standage, "Why Napster Matters," Prospect (October), pp. 50-53, 2000 .

[7] F. Pereira, I. Burnett, "Universal Multimedia Experiences for Tomorrow," Signal Processing Magazine, IEEE, Volume: 20, Issue: 2, March 2003, pp. $63-73$.

[8] L. Rong and I. Burnett, "Dynamic resource adaptation in a heterogeneous peer-to-peer environment," Consumer Communication and Networking Conference (CCNC 2005), January, 2005.

[9] S. Saroiu, P. K. Gummadi, and S. D. Gribble, "A Measurement Study of Peer-to-Peer File Sharing Systems," in proceeding of the Multimedia Computing and Networking, January, 2002.

[10] L. Rong and I. Burnett, "Improved Dynamic Adaptation-based Peer-toPeer System with Locality-based Clustering," Automated Production of Cross Media Content for Multi-channel Distribution Conference (AXMEDIS 2005), November, 2005.

[11] [Online]. Available: http://freenet.sourceforge.net/ 